\title{
A Survey of Radio Resource Management for Spectrum Aggregation in LTE-Advanced
}

\author{
Haeyoung Lee, Seiamak Vahid, and Klaus Moessner
}

\begin{abstract}
In order to satisfy the requirements of future IMTAdvanced mobile systems, the concept of spectrum aggregation is introduced by 3GPP in its new LTE-Advanced (LTE Rel. 10) standards. While spectrum aggregation allows aggregation of carrier components (CCs) dispersed within and across different bands (intra/inter-band) as well as combination of CCs having different bandwidths, spectrum aggregation is expected to provide a powerful boost to the user throughput in LTE-Advanced (LTE-A). However, introduction of spectrum aggregation or carrier aggregation (CA) as referred to in LTE Rel. 10, has required some changes from the baseline LTE Rel. 8 although each CC in LTE-A remains backward compatible with LTE Rel. 8. This article provides a review of spectrum aggregation techniques, followed by requirements on radio resource management (RRM) functionality in support of CA. On-going research on the different RRM aspects and algorithms to support CA in LTEAdvanced are surveyed. Technical challenges for future research on aggregation in LTE-Advanced systems are also outlined.
\end{abstract}

Index Terms-Carrier aggregation, Spectrum Aggregation, Radio Resource Management, Scheduling

\section{INTRODUCTION}

$\mathbf{I}$ $\mathrm{N}$ order to meet the growing demand for high-speed and diverse wireless broadband services, the IMT-Advanced (IMT-A) requirements have established a minimum support for $1 \mathrm{Gbps}$ and $500 \mathrm{Mbps}$ peak rates for downlink (DL) and uplink (UL), respectively [1]. In order to fulfil these challenging requirements, one key feature is the support for wider bandwidths ( $40 \mathrm{MHz}$ mandatory, and up to a maximum of $100 \mathrm{MHz}$ being optional) [2].

IMT Bands, i.e. the candidate frequency bands for IMTAdvanced, identified at World Radio Conferences (WRCs) are non-continuous, and some of them are less than 100 $\mathrm{MHz}$ [3] as shown in Fig. 1. Specifically, the amount of contiguous transmission bandwidth for an operator in a certain geographical area is limited while the extent of available spectrum resources differs depending on the country, with most of the spectrum spread out over different frequency bands and with different bandwidths [4]. In order to meet both the requirement on transmission bandwidth and the utilization of IMT bands, all IMT-Advanced candidate technologies are expected to support spectrum aggregation, within either contiguous or discontinuous spectrum bands [5].

Spectrum aggregation (or carrier aggregation) was introduced by $3 \mathrm{GPP}$ in its new LTE-Advanced standards, a candidate radio interface technology for IMT-Advanced systems.

Manuscript received November 30, 2012; revised May 21, 2013; and revised July 21, 2013.

The authors are with the Centre for Communication Systems Research (CCSR), University of Surrey, Guildford, GU2 7XH, U.K., (e-mail: Haeyoung.Lee, S.Vahid, K.Moessner@surrey.ac.uk).

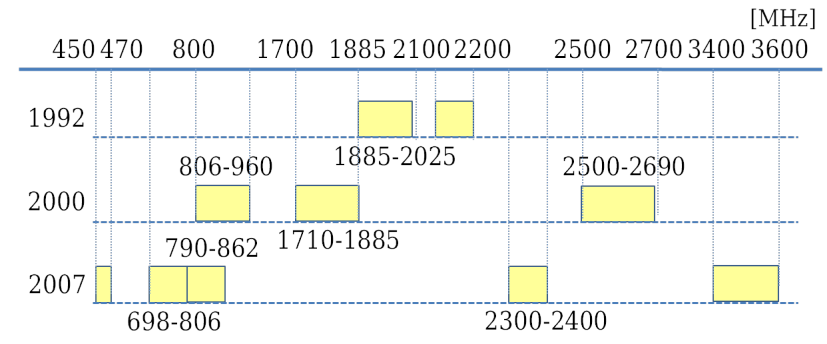

- Regional base: 698-960 MHz for Region 2, 790-960 MHz for Region 1\&3 - Country base : for some countries in Region $1 \& 3$

Fig. 1. Identified IMT Bands

However, the concept of carrier aggregation (CA) is not $t$ new. It has already been deployed in HSPA based c systems, under the name Dual Carrier HSPA (DC-H to aggregate two adjacent carriers in the DL/UL [6. carriers must be contiguous and in the same spectrun Unlike DC-HSPA, however, carrier aggregation in has extended the concept to introduce aggregation ( contiguous spectrums in different spectrum bands [7 or more component carriers (CCs) of different bandwi different bands can be aggregated (up to $100 \mathrm{MHz}$ w CCs of $20 \mathrm{MHz}$ ) to support wider transmission ban between the E-UTRAN NodeB (eNB) and the user equ (UE) [8].

LTE-Advanced supports the same range of $\mathrm{CC}$ band (1.4 MHz, $3 \mathrm{MHz}, 5 \mathrm{MHz}, 10 \mathrm{MHz}, 15 \mathrm{MHz}$, and 20 that are supported in LTE Rel. 8. While LTE-A su bandwidth extension by aggregating CCs, subject to spe availability and the UE's capability [10], CC backward patibility has been a requirement in LTE-Advanced fron outset as shown in Fig. 2. With each CC in LTE-A b

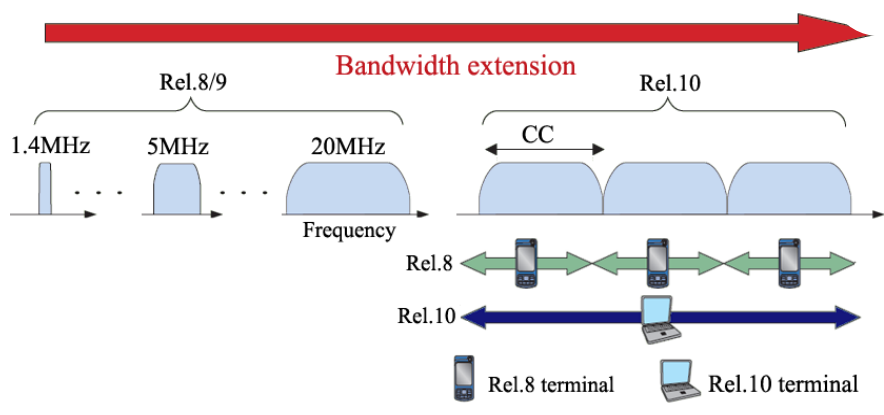

Fig. 2. Bandwidth Extension by Carrier Aggregation [9] 


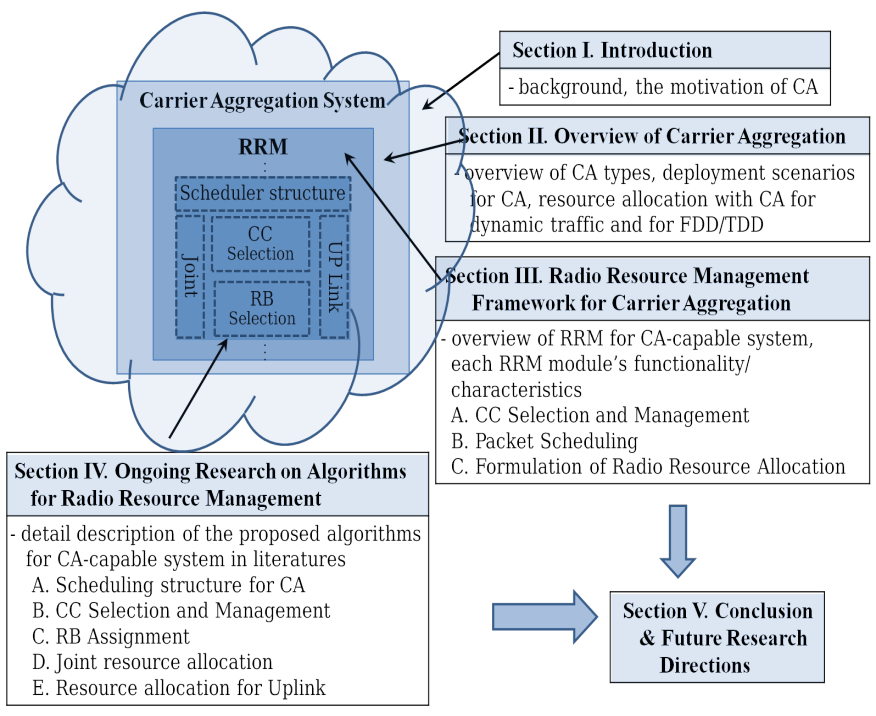

Fig. 3. Structure of this survey paper

LTE Rel. 8 compatible, carrier aggregation allows operators to migrate from LTE to LTE-Advanced while continuing to provide services to any LTE users. This is made possible since the eNB and Radio Frequency (RF) specifications associated with LTE Rel. 8 remain unchanged in LTE-A [11]. By reusing the LTE design on each of the CCs, both implementation and specification efforts are minimized [12]. However, the introduction of CA for LTE-Advanced has required the introduction of new functionalities and modifications to the link layer and radio resource management (RRM) [13]. In this paper, we focus on the RRM framework to support CA functionality and present a survey of existing literature on RRM schemes for CA in LTE-A.

The paper is organized based on the structure shown in Fig. 3. An overview of the CA scheme in LTE-Advanced is provided in Section II. This is followed, in Section III, by a discussion on the RRM scheme for CA. In Section IV, an overview of current research on resource allocation schemes for downlink and uplink is provided. Finally, in Section V, we point out future research directions based on open challenges and provide conclusions.

\section{Overview of CARrier Aggregation}

Three different types of carrier aggregation are identified according to the way in which CCs are arranged [9], [14]. This is illustrated in Fig. 4.

- Intra-band contiguous CA: In this case, a contiguous bandwidth wider than $20 \mathrm{MHz}$ is used for LTE-Advanced. The spacing between centre frequencies of contiguously aggregated CCs is forced to be a multiple of $300 \mathrm{kHz}$. This may be a less likely scenario given frequency allocation today, but it can be applied for example, to broadband allocation in the $3.5 \mathrm{GHz}$ band.

- Intra-band non-contiguous CA: When the contiguous spectrum blocks are not available for aggregation, multiple non-contiguous CCs belonging to the same band can be used [15].

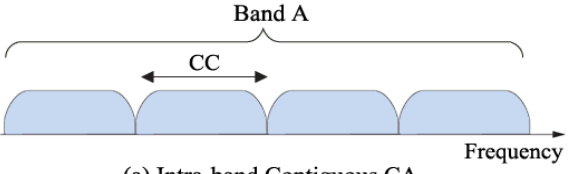

(a) Intra-band Contiguous CA

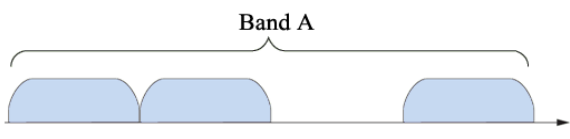

(b) Intra-band non-contiguous CA

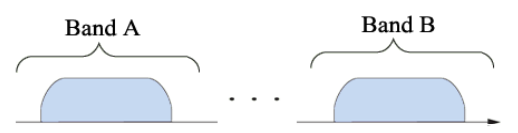

(c) Inter-band non-contiguous CA

Fig. 4. Three types of spectrum aggregation

- Inter-band non-contiguous CA: In this case, communications are performed using different frequency bands, such as the $2 \mathrm{GHz}$ band and the $800 \mathrm{MHz}$ band. With this type of aggregation, robustness to mobility can potentially be improved by exploiting different radio propagation characteristics of different bands.

The support for both contiguous and non-contiguous CA of CCs with different bandwidths offers significant flexibility for efficient spectrum utilization, and gradual reframing of frequencies previously used by other radio access systems. However, from the physical layer perspective, it is easier to implement contiguous CA without making many changes to the physical layer structure of LTE system [16]. In order to achieve contiguous CA for an LTE-Advanced UE unit, it is possible to use a single fast Fourier transform (FFT) module and a single RF unit while providing backward compatibility to the LTE systems. For the non-contiguous CA, in most cases, multiple RF chains and FFT modules will be required. From the perspective of resource allocation and management, contiguous CA is also easier to implement. Different CCs will exhibit different propagation path loss and Doppler shift which will affect the system performance, as shown in [16]. For example, Doppler shift influences the gains from frequency domain packet scheduling within a CC [13]. In LTE-Advanced, for the UL, the focus is currently on intraband non-contiguous $\mathrm{CA}$, due to difficulties in defining RF requirements for simultaneous transmission on multiple CCs with large frequency separation, considering realistic device linearity constraints. For the DL, however, both intra- and inter-band cases are considered in Rel. 10, while specific RF requirements are being developed [17].

Generally, carrier aggregation systems are deployed to improve user data rates rather than spectral efficiency, although operation over multiple carriers can also provide some level of enhanced interference control [14]. The deployment scenarios shown in Fig. 5 [18] were considered during the design of LTE-Advanced carrier aggregation, exemplified with two component carriers at frequencies of F1 and F2. In one of the most typical deployment scenarios, the eNB antennas are collocated and have the same beam directions/patterns for different CCs. 

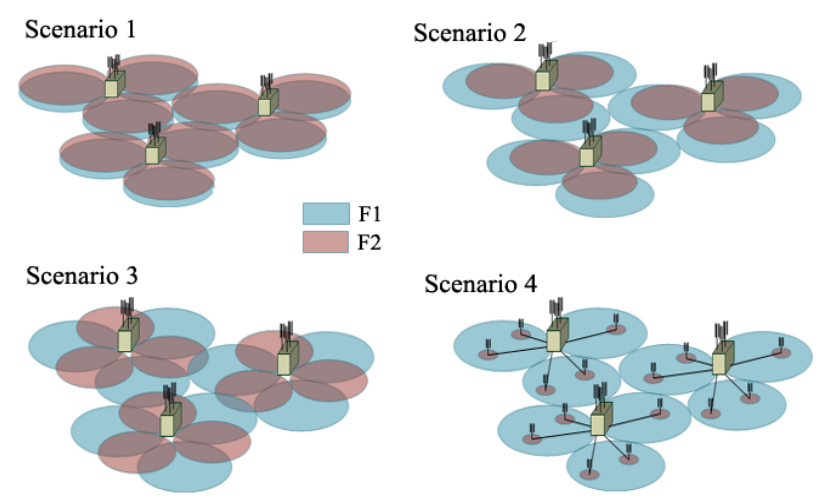

Fig. 5. Carrier aggregation deployment scenarios (F2>F1) [18]

If the CCs are at the same band or the frequency separation is small, this would lead to nearly the same coverage for all CCs as shown in scenario 1. Large frequency separation between CCs would lead to the scenario 2 where the coverage of CCs is different. Even in the same band CCs may be deployed at eNBs with different transmit power levels to provide different coverage footprints for intercell interference management purposes [14]. In either case, CA allows higher user throughput at places where coverage of CCs overlap. In scenario 3, different beam directions or patterns are used for different CCs to shift the beams across carriers, and in doing so to improve throughput at the cell edges. In scenario 4 , one $\mathrm{CC}$ (usually a low frequency) provides macro coverage while other CC (usually a high frequency) is utilized to absorb traffic from hotspots using Radio Remote Head (RRH) units. RRHs are connected via optical fiber to the eNB, thereby allowing the aggregation of CCs between the macrocell and RRH cell based on the same CA framework for collocated cells. Such deployments allow the operators to improve system throughput by using low-cost RRH equipment [19].

The most efficient deployment scenario can be decided based on many factors, such as whether the service environment is urban, suburban, or rural; and whether there are hot spots in the area. A likely near-term scenario is that an existing deployment on a legacy frequency band (e.g., $2 \mathrm{GHz}$ ) is used to provide sufficient coverage across the service area, and new bands (e.g., $3.5 \mathrm{GHz}$ ) are used to serve traffic in a more cost-effective manner. CA is expected to allow for such flexible use of spectrum in heterogeneous networks, depending on the operator's needs [20]. In all cases of carrier aggregation, different LTE-Advanced UEs even within a single eNB will be configured with different number of CCs [21]. Depending on the UE's capability and deployment scenarios, CCs involved in the configured set of serving cells may be contiguous or non-contiguous.

When an LTE-Advanced UE first establishes or reestablishes radio resource control (RRC) connection (with eNB), only one CC is configured (for DL and UL, respectively), referred to as the primary CC (PCC) corresponding to the primary serving cell (PCell). That is, the DL CC is designated as the DL primary CC (DL PCC), and the corresponding UL CC is designated as the UL PCC. Then, depending on

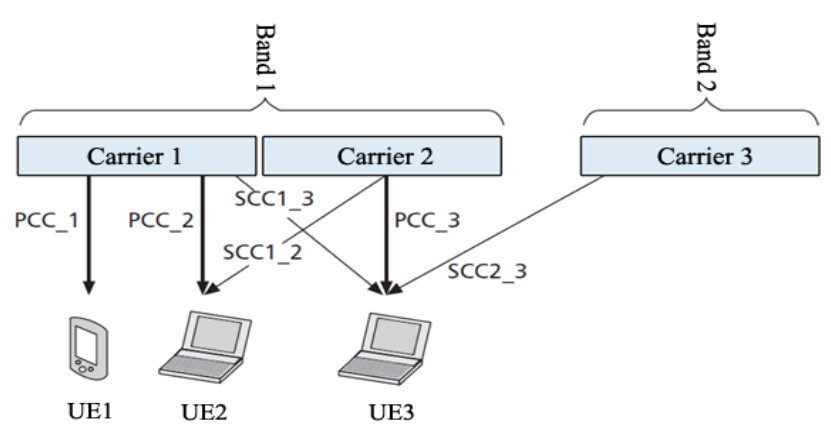

Fig. 6. Configuration of different number of CCs [14]

traffic load and quality of service $(\mathrm{Q} o \mathrm{~S})$ requirements, the UE can be configured with one or more additional CCs, called secondary CCs (SCCs) for secondary serving cells (SCells). The DL and UL CCs are called the DL and UL secondary CCs (SCCs), respectively [13], [22]. The usage of DL/UL SCCs by the UE is also configurable by the eNB. The PCC/SCCs configuration is UE-specific and can be different for different UEs served by the same eNB. In other words, different users may not necessarily use the same CC as their PCC and a CC at an eNB may be the PCC for one UE and serve as a SCC for another UE as shown in Fig. 6.

The PCC can be regarded as the anchor $\mathrm{CC}$ for the user and is thus used for basic functionalities such as radio link failure monitoring and etc. The SCCs convey only the dedicated signalling information, PDSCH (physical DL shared channel), PUSCH (physical UL shared channel), and PDCCH (physical DL control channel). Meanwhile, LTE-A supports cross-carrier scheduling which means PDCCH transmitted from a particular $\mathrm{CC}$ may contain the scheduling information on other CCs as well as its own CC [23]. That is, the DL control channel of PCC (subject to small interference) can be used in order to schedule DL and UL resource on the SCCs (subject to large interference). This method is expected to be useful for interference management for control channels in heterogeneous networks [24] and enable load balancing across different cell layers [25]. The DL and UL PCCs should therefore be robust, and are typically chosen such that they provide the most ubiquitous coverage and/or best overall signal quality (i.e. based on measurements of reference signal received power (RSRP) or reference signal received quality (RSRQ) [18]) [22]. As UE moves within the geographical area served by an eNB, the PCC may be changed to correspond to the CC of the best signal quality. PCC change can also be performed by the eNB based on other considerations such as load balancing [14]. Depending on such elements as the buffered data amount, required QoS, and carrier loading, the use of DL SCCs could be dynamically activated and deactivated. Since the amount of the signalling is reduced for the deactivated SCCs, the battery power consumption of UEs can be reduced by activating/deactivating SCCs dynamically.

For LTE with FDD, DL/UL carriers are always paired with options for defining the frequency duplex distance and bandwidth through system information signalling. However, in 
LTE-Advanced, asymmetric CA is supported in two directions; that is, the numbers of aggregated CCs in two directions can be different, thus improving spectrum efficiency. In this context, UE configured with CA may need to interact with eNB on unequal numbers of DL and UL CCs when the usage of UL SCCs of some of the SCells is not configured. Note that DL CCs may be linked to UL CCs with duplex distances different than those defined in LTE. The asymmetric CA could cause ambiguity in downlink component carrier selection, because it is difficult for an LTE-Advanced eNB to know the CC to which UE anchors in downlink. The linking between uplink and downlink configured CCs is signalled to the UE with higherlayer signalling [13]. In time-division duplex (TDD) mode, asymmetric CA can also be achieved by adjusting the ratio of allocated time slots for uplink and downlink transmissions. This scheme simplifies the resource allocation relationship between the uplink and downlink channels [16].

\section{Radio Resource MANAGEMEnt Framework For CARrier Aggregation}

The RRM framework for LTE-Advanced retains many similarities with that of LTE. With carrier aggregation, however, it becomes possible to schedule a user on multiple component carriers simultaneously each of which may exhibit different radio channel characteristics. Supporting multi-CC operations introduces some new challenging issues in radio resource management (RRM) framework for LTE-Advanced systems [26]. In this section, the distinctions between LTE-Advanced systems and LTE are highlighted from the RRM perspective.

The RRM structure for a carrier aggregation system is illustrated in Fig. 7. Admission control in LTE-A is performed by the eNBs prior to establishment of new radio bearers, and the QoS parameters are configured. The QoS parameters are the same for LTE and LTE-Advanced, and are thus CCindependent. Then, based on QoS requirements and traffic loads, the eNB configures a set of CCs for users and resource block scheduling is carried out via multiplexing of multiple users on each CC. In order to allow for backward compatibility so LTE and LTE-Advanced users can co-exist, it has been decided to use independent layer-1 transmissions, which contain Link Adaptation (LA) and Hybrid Automatic Repeat request (HARQ) etc, per CC, in line with the LTE assumptions [10], [27].

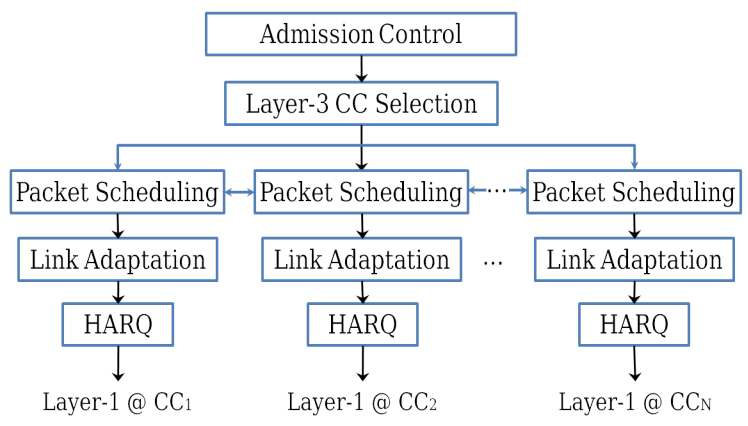

Fig. 7. RRM structure of LTE-Advanced with Carrier Aggregation [27]
Independent link adaptation per $\mathrm{CC}$ helps to optimize transmission on different $\mathrm{CCs}$ according to the experienced radio conditions. By setting different transmit powers for individual CCs, CCs could provide different levels of coverage [13]. Especially, in the case of inter-band CA, since the radio channel characteristics such as propagation, path loss, building penetration loss, and doppler shift, vary significantly at different frequency bands, selecting different transmission parameters including modulation scheme, code rate, and transmit power per $\mathrm{CC}$ is expected to be useful to further improve user QoS [28]. While aggregation capability provides the flexibility to deal with bandwidth extensions required to achieve high data rates and wider coverage [29], multiple component carrier resource allocation and adaptive adjustment of transmission parameters for different CCs should be jointly considered [30]. In addition, the independent layer-1 transmissions incur a large amount of transmission overhead due to uplink signalling: for channel-aware packet scheduling and link adaptation, a UE has to feedback the channel quality indicator (CQI), which contains information about the channel quality, and acknowledgement/non-acknowledgement (ACK/NACK) per CC, which indicates whether or not transmission has been successful for the independent layer-1 transmissions on each CC. If users are allocated multiple CCs, the uplink signalling from users will contribute to a large amount of uplink overhead. Several techniques to reduce this overhead at different layers are described and evaluated in [31].

\section{A. CC Selection and Management}

$\mathrm{CC}$ selection, to assign multiple CCs to users, is the new RRM functionality introduced in LTE-Advanced. For CC selection, UE's information such as the QoS requirements and terminal capability can be exploited as well as the measured information including the overall traffic level, the traffic load per CCs, and the channel quality information from UEs. The overall framework for the $\mathrm{CC}$ configuration could be illustrated in Fig. 8.

For determining the number of required $\mathrm{CCs}$ for the user, QoS parameters such as the QoS class identifier (QCI), guaranteed bit rate (GBR) for GBR bearers, and aggregated maximum bit rate (AMBR) for non-GBR bearers could be useful. As an example, for users with a voice over IP (VoIP) call or a streaming connection with moderate GBR requirements, a single CC can be assigned to satisfy the users' QoS requirements. For users having best effort traffic, the AMBR

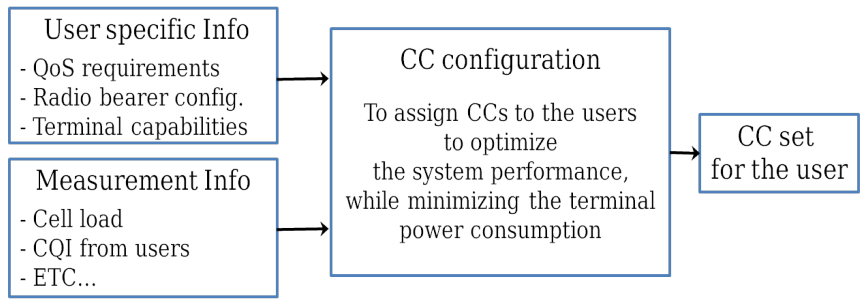

Fig. 8. Overview of $\mathrm{CC}$ configuration functionality including possible input parameters [13] 
requirements can be used to estimate the suitable $\mathrm{CC}$ set size for them.

For optimal system performance, it is desirable to have approximately equal load on different CCs, so own-cell load information (including load per $\mathrm{CC}$ ) is needed for CC configuration as well. Unbalanced traffic load across the CCs will lead to under-utilization of spectrum resources [32]. Actually, assigning all CCs to users maximizes the trunking efficiency and the frequency domain packet scheduling gain [33].

At low traffic levels, the performance gain from using multiple CCs by CA over using a single CC is significant in terms of the average user throughput. Simulation studies in [33] show that assigning all CCs to users achieves 100-300\% higher user throughput/coverage than assigning a single CC per user. However, as the traffic increases, the gain diminishes [13] and different load balancing methods achieve roughly similar performance. Since increase of the number of CCs a user has to receive (i.e. increase of bandwidth it needs to process) leads to higher signal processing complexity and power consumption, only a small number of CCs should be configured at high traffic loads [33].

Especially for uplink transmission, the maximum transmission power becomes a constraint [26]. In the uplink, increasing the bandwidth does not necessarily result in an increase of data rates if a UE reaches its maximum transmission power. In addition, when a UE is transmitting over multiple CCs simultaneously, peak-to-average power ratio (PAPR) increases and this results in effective reduction of the UE maximum transmission power [34] in order to maintain operation in the linear region of the power amplifier. Especially for powerlimited users that experience unfavourable channel conditions, it is not a good idea to always allocate multiple CCs [26], [35]. On top of the increased PAPR, there are also RF issues (e.g. inter-modulation effects) which result in an additional reduction of the maximum UE transmission power. While several factors affect the amount of power reduction [26], the power reduction is modelled by the power back-off and its impact on the uplink performance of $\mathrm{CA}$ is investigated in [35]. The increase in power back-off causes the degradation of the average throughput of LTE-Advanced UEs by reducing the maximum UE transmission power, which results in decreasing the probability of being assigned to multiple CCs for LTEAdvanced UEs. Due to the transmission power constraint, the CC selection scheme for UL plays a more important role in optimizing the system performance with CA than that for DL from RRM perspective.

In LTE-Advanced system with coexisting LTE-Advanced and legacy LTE UEs, fairness can be an issue for CC scheduling. Since the LTE-Advanced UEs will be scheduled on more CCs than the LTE users, the LTE UEs achieve much lower throughput than the LTE-Advanced UEs [36]. Since the coverage throughput is taken as the $5 \%$ worst user throughput, bad coverage performance could indicate a low LTE user throughput.

As already mentioned in Section II, the eNB firstly allocates the user with a PCC of the best signal quality to provide the most ubiquitous coverage. After allocating PCC based on the measurement report (MR) of CCs' signal quality, eNB makes decision on whether additional SCCs are needed or not, considering other aspects such as the buffered data amount, user's QoS requirement and carrier loading [22]. SCCs can be dynamically activated and deactivated independently via MAC. The configured SCCs are by default de-activated, so they have to be explicitly activated before being scheduled. However, the PCC for a user is always assumed to be activated and is therefore not subject to any de-activation procedures [37].

The mobility management of CC, i.e. inter/intra-frequency handover, $\mathrm{CC}$ addition, and $\mathrm{CC}$ removal, enables control over coverage and overall signal quality in deployments supporting carrier aggregation. However, these operations can increase the signalling overhead associated with RRC configuration. As CCs are dynamically changed depending on the radio conditions, the frequency of RRC configuration is increased, leading to increased RRC signalling although the SINRs are enhanced [38]. A UE in power limited situation may experience outage due to lack of power to transmit the required feedbacks corresponding to all configured CCs [31]. Thus, it is important to reduce the signalling overhead by avoiding excess handovers and by deactivating SCCs dynamically. While the signalling overhead is also influenced by the scenario, the carrier aggregation of $\mathrm{CCs}$ with different coverage increases the RRC signalling overhead. Different CC management policies that offer different RRC signalling overheads are investigated in [38].

How best to assign the $\mathrm{CCs}$ to each user according to its carrier capability and the circumstances, as well as how to multiplex multiple users in each CC [22] [39], remains one of the key issues in the design of resource management schemes for CA-based systems.

\section{B. Packet Scheduling}

After multiple CCs are configured, packet scheduling (PS) is performed across configured CCs [22]. The PS aims to benefit from multi-user frequency domain scheduling diversity by prioritizing the allocation of resource blocks (PRBs) to users that experience good channel quality. Although out of scope of specifications, the PS functionality for LTE-Advanced with CA remains very similar to the PS scheme used in LTE Rel-8, except that the LTE-Advanced PS is allowed to schedule users across multiple CCs which are configured and activated for UEs [13]. Similar to the LTE PS framework, the smallest frequency domain scheduling resolution within each CC in LTE-A, is a PRB of 12 subcarriers, constituting an equivalent bandwidth of $180 \mathrm{kHz}$. One PRB also corresponds to a subframe in the time domain, with a Transmission Time Interval (TTI) of $1 \mathrm{~ms}$.

While LTE-Advanced relies on independent transport blocks, link adaptation, and HARQ per CC, such independent operation per $\mathrm{CC}$ opens up various implementation options for the scheduler. As an example, scheduling could be done either jointly across multiple CCs or independently for each CC [13] (as shown in Fig. 9). When joint scheduling is used, extra complexity is introduced by either a more sophisticated scheduler operating across multiple CCs or exchanging information 
between independent schedulers. Compared with independent scheduling, resource allocation across multiple CCs, increases scheduling load of eNB as well as uplink overheads. However, resource allocation across multiple $\mathrm{CCs}$ has better performance by enabling load balancing between carriers and thus fairness is improved [32], [40].

Design requirements for packet schedulers in LTEAdvanced system with CA, should address: 1) the need to handle the packet scheduling in multiple CCs environments, 2) the need to support required QoS for various traffic types, 3) the need for high system throughput, and 4) maintaining fairness among users (LTE-Advanced and LTE UEs), as identified in [41].

\section{Formulation of Radio Resource Allocation}

The radio resource allocation problem for co-existing LTEAdvanced and LTE UEs, can be formulated as described in [39]. Among the $K$ users, there are $K_{1}$ LTE UEs and $K_{2}$ LTE-Advanced UEs. Let $\mathcal{K}_{1}$ and $\mathcal{K}_{2}$ be the set of LTE users and the set of LTE-Advanced users, i.e. $\mathcal{K}_{1}=\left\{1,2, \ldots, K_{1}\right\}$, $\mathcal{K}_{2}=\left\{1,2, \ldots, K_{2}\right\}$, respectively. The set of the total user is $\mathcal{K}=\{1,2, \ldots, K\}$. It is assumed that there are $M \mathrm{CCs}$ in the system, denoted by the set $\mathcal{M}=\{1,2, \ldots, M\}$. Define $\alpha_{k, i} \in\{0,1\}$ to be the CC allocation indicator, where $\alpha_{k, i}=$ 1 indicates that the $i^{\text {th }} \mathrm{CC}$ is assigned to the $k^{\text {th }}$ user, and otherwise $\alpha_{k, i}=0$ in Eq.(2). For LTE users, only one CC can be assigned. For LTE-Advanced UEs, the multiple CCs can be assigned as shown in Eq.(3). Let all CCs of the same bandwidth have $N$ resource blocks (RBs). Of course, in the case of CCs of the different bandwidth, the number of RBs of each $\mathrm{CC}$ will be different. Let $\beta_{k, i, j} \in\{0,1\}$ be the RB allocation indicator, where $\beta_{k, i, j}=1$ represents that the $j^{\text {th }}$ $\mathrm{RB}$ in the $i^{t h} \mathrm{CC}$ is allocated to the $k^{t h}$ user, and otherwise, $\beta_{k, i, j}=0$. In order to avoid co-channel interference, each $\mathrm{RB}$ should be assigned to at most one user in each frame as described in Eq.(5).

$$
P=\max \sum_{k} \sum_{i} \sum_{j} u_{k, i, j} \cdot \alpha_{k, i} \cdot \beta_{k, i, j}
$$

subject to

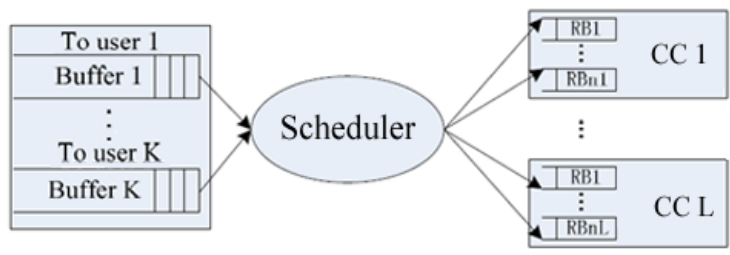

(a) Joint Queue Scheduler (JQS)

$$
\begin{gathered}
\alpha_{k, i} \in\{0,1\}, \forall k \in \mathcal{K}, \forall i \in \mathcal{M} \\
\sum_{i} \alpha_{k, i} \leq 1, \forall k \in \mathcal{K}_{1} \text { and } \sum_{i} \alpha_{k, i} \leq M, \forall k \in \mathcal{K}_{2} \\
\beta_{k, i, j} \in\{0,1\}, \forall k \in \mathcal{K}, \forall i \in \mathcal{M}, \forall j \in \mathcal{N} \\
\sum_{k} \beta_{k, i, j} \leq 1, \forall i \in \mathcal{M}, \forall j \in \mathcal{N}
\end{gathered}
$$

In Eq.(1), $u_{k, i, j}$ represents the utility of the $k^{t h}$ user on the $j^{\text {th }} \mathrm{RB}$ of the $i^{\text {th }} \mathrm{CC}$ in each time frame. As a constrained optimization model, the joint resource allocation problem, $P$, has the objective of maximizing the system utility. The formulation in Eq.(1) represents a non-linear integer programming problem. Since the computational complexity depends on the value of $K, N$ and $M$, an exhaustive search over all combination could be impractical for the large values of $K, N$ and $M$ [39]. To reduce the computational complexity, the resource allocation with aggregation could be decomposed into two sequential steps, i.e. CC selection followed by RB assignment on each CC [22], [32], [36], [42]. In the first step, the eNB employs CC selection to assign users on proper CCs. Once the users are assigned onto certain CC(s), the assignment of RBs belonging into the $\mathrm{CC}$ is carried out [39].

\section{Ongoing Research on Algorithms for Radio RESOURCE MANAGEMENT}

In this section, after providing a general overview of scheduling structures for CA, algorithms for CC selection and $\mathrm{RB}$ scheduling proposed in the literature are reviewed. In most of the existing works on resource allocation for CA, only the downlink of the wireless system is considered since higher application throughputs are required in the downlink rather than in the uplink [40]. Whilst there are a few similarities between radio resource management for DL and UL, the maximum transmission power is a constraint only for UL. The literature survey on the resource allocation algorithms for UL will be presented at the end of this section.

\section{A. Scheduling structure for $C A$}

For resource scheduling in CA based systems, two different scheduler structures are proposed in [40]: Joint Queue Sched-

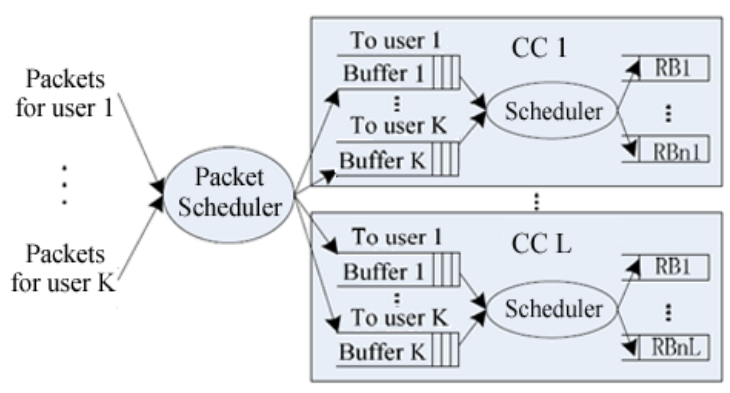

(b) Disjoint Queue Scheduler (DQS)

Fig. 9. An example of the scheduling structure for CA [40] 
uler (JQS) and Disjoint Queue Scheduler (DQS) as illustrated in Fig. 9. In the structure of JQS, shown in Fig. 9(a), each user has only one queue for all CCs, and the CCs share the joint queue of each user. The shared scheduler maps the users' traffic to the RBs on all CCs. That is, the JQS approach combines all $\mathrm{CCs}$ as one carrier to allocated RBs [39]. Although one user's data may only be transmitted on some of the CCs, JQS requires each user to be able to receive a signal from all CCs simultaneously and continuously. It largely increases the signal processing complexity and the power consumption of the UEs [40], [43], [44]. Considering that the bandwidth of the LTE-Advanced system is wide, the $J Q S$-based implementation can lead to increased complexity [32].

As mentioned earlier, the aggregated spectrum allocation could be decomposed into $\mathrm{CC}$ selection and $\mathrm{RB}$ assignment phases to reduce the computational complexity. For two-step scheduling, Disjoint Queue Scheduler (DQS) is illustrated in Fig. 9(b). In this scheme, each user has one traffic queue on each CC. Through two-layer scheduling, the traffic packets of users are assigned onto certain $\mathrm{CC}(\mathrm{s})$ and the assignment of $\mathrm{RBs}$ in each $\mathrm{CC}$ is then carried out.

When comparing the performance of two scheduler schemes, the performance of $D Q S$ is found to be inferior than that of $J Q S$ in two aspects: lower spectral efficiency and unsaturated resource utilization. While in $J Q S$, a user data packet can use all RBs on all CCs, a data packet in $D Q S$, can be transmitted by RBs on only selected CCs, which are a subset of all CCs. Thus, the $D Q S$ scheme results in lower frequency selective gains. In addition, the automatic traffic load balancing over CCs is expected in JQS while $D Q S$ may result in unbalanced loading across CCs [40]. Thus, $D Q S$ cannot fully utilize the resources. JQS is considered as the optimal scheduler for the LTE-Advanced system with CA, at the cost of high complexity [32].

In [32], [40], [43], the performance of the CA scheduling of $J Q S$, is analyzed and compared with that of Independent Carrier Scheduling (ICS) scheme (i.e. non-CA scheduling) in the scenario of two CCs belonging to the same band. The main characteristic of ICS is that each user can access only one CC until the end of its traffic, and this user can utilize RBs on this CC. In the ICS scheme, the importance attached to traffic load balancing over multiple CCs is evident. When allocating two CCs to ten users, it was found that more symmetrical user assignments on CCs can result in higher throughputs. Since load balancing impacts fairness between users, in the case of unbalanced load distribution on $\mathrm{CCs}$, the throughput of users on the more crowded carrier is reduced. However, the CA scheduling based on JQS shows better fairness among the users by automatically balancing the load.

For different traffic patterns and loads, the performance of the aforementioned scheduling schemes are further investigated in [40].

As demonstrated in Fig. 10(a), under the full buffer model, simulation results show that the CA scheduling based on JQS always produces higher throughput than ICS with various combinations of the traffic load (the number of users) on CCs. The analysis indicates that the capacity gains are due to joint scheduling and improved multi-user diversity gain.

For the bursty traffic model, the gains for the CA-based scheduling (JQS) over ICS scheme are reported and shown for two CCs (See in Fig. 10(b).). When the traffic load is not too high, JQS can provide about twice the average burst rate than ICS at the same number of users per sector. Furthermore, the ICS scheme can support exactly twice the number of users per sector supported by one single carrier at the same average burst rate. In [45], an analytical study shows that the performance of JQS is $L$ times better than ICS in terms of the average user throughput, where $L$ is the number of CCs in the systems. Besides providing better user throughput, the scheduling scheme of $J Q S$ offers substantial benefits in load balancing, packet latency and burst rate.

When comparing the two scheduling schemes of JQS and $D Q S$, it is observed that the average burst rate supported in $D Q S$ is always lower than that of JQS as shown in Fig. 10(c). The gain in average data rate of $J Q S$ over $D Q S$ decreases with the number of users per sector due to decrease in difference in frequency selective gains. In addition, it is shown that $D Q S$ is inefficient especially when the packets are large and sparse [40].

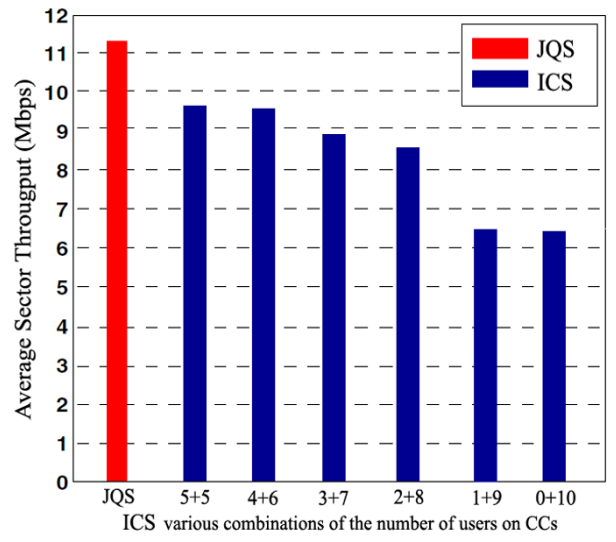

(a) Full Buffer (JQS vs. ICS)

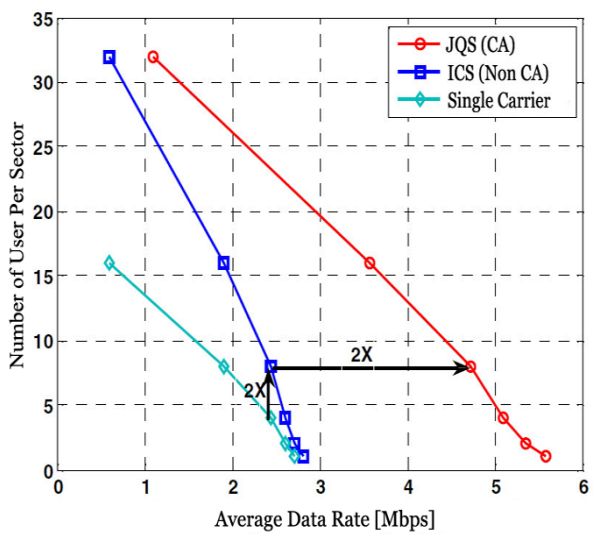

(b) Bursty Traffic (JQS vs. ICS)

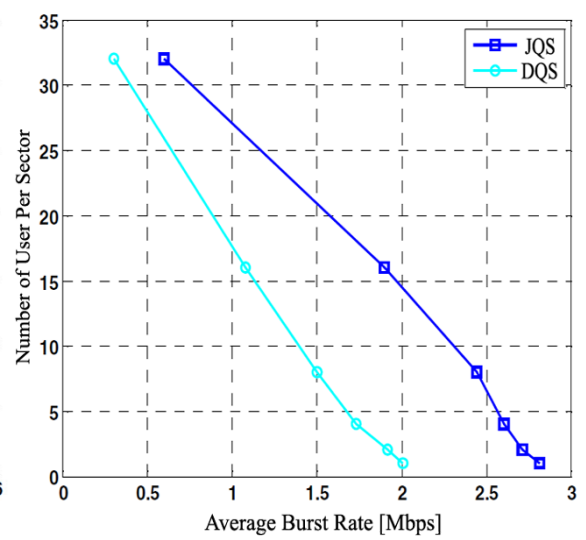

(c) Bursty Traffic (JQS vs. DQS)

Fig. 10. The performance comparison of scheduler schemes for different traffic patterns [40] 
While scheduling performance gains of the CA scheme over non-CA scheme have been investigated in the literature, there is concern that channels wider than $3 \mathrm{MHz}$ could have similar spectral efficiencies (under the full buffer traffic model) for two reasons: 1) overheads due to the control signalling become small as a proportion of total resources, 2) fewer opportunities for additional frequency diversity given realistic channel delay spreads. However, it is highlighted in [46] that trunking gains in wider bandwidth systems may produce some improvements in spectral efficiency when high user throughputs are being targeted. In order to maximize the data rates and to optimize the spectral efficiency, it is concluded in [47] that the system should be deployed using as large a bandwidth as possible.

Since the unbalanced traffic loading across multiple CCs leads to performance degradation, advanced rules for user allocation to CCs are proposed in [26] to help balance the loading across CCs. However, it is impossible to totally avoid the resource wastage even when the traffic is balanced well over $\mathrm{CCs}$ through application of the advanced $\mathrm{CC}$ selection rules recommended in [44]. In order to overcome this challenge, two schemes are proposed in [32], [44]: 1) CC switching, and 2) CC coupling.

The first scheme was originally proposed for ICS. While the $\mathrm{CC}$ allocated to a user is not changed in ICS, the CC for one user can be exchanged at the burst level as proposed in [32]. It is shown that decreasing the number of bursts (i.e. traffic dispatching granularity) during the period when the same CC is utilized without exchanges leads to better traffic load balancing and improvements in resource utilization.

In [44], the concept of CC coupling is proposed. If the CCs are in different working states (busy/idle), the users connecting to the busy CCs can be temporarily allowed to transmit on those idle CCs for a period of time through CC coupling. If the idle CCs become busy, the coupling is cut at once. To do this, at the beginning of each frame of a user, the eNB needs to couple the idle and busy CCs and send the corresponding coupling information to the UEs. Since the idle CCs help the busy CCs, the resource utilization is increased. It is shown that average delay performance of the ICS scheme becomes almost the same as that of the JQS scheme by allowing CC coupling at the frame level, regardless of the carrier scheduling scheme and the traffic load. However, there remains challenges associated with: 1) CC switch delay, and 2) development of the efficient coupling methods for multiple CCs of different

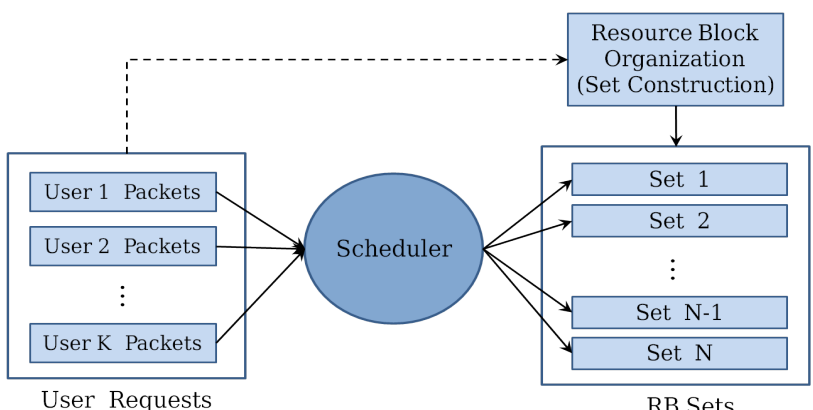

Fig. 11. The scheduling scheme using resource block organization [48]

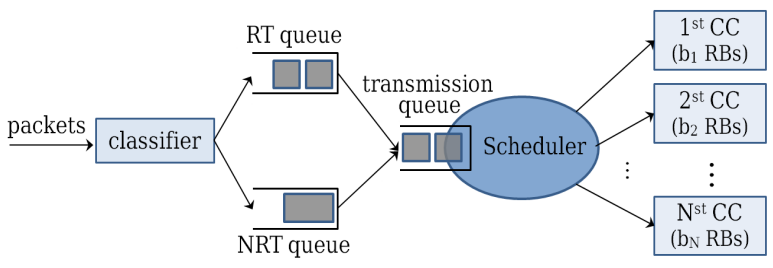

Fig. 12. The scheduling scheme for real-time traffics [41]

BWs belonging to different bands.

Since the scheduling delay is an important design constraint, scheduling structures are proposed to minimize the delay in [41] and [48]. In order to reduce scheduling times, RB grouping method is proposed in [48]. Since the scheduling delay is more sensitive for real-time traffic, the scheduling scheme supporting higher priority for real-time packets is proposed in [41].

When the scheduler for the CA system uses an individual assignment strategy for available RBs, the time required by the scheduler to assign total $M$ RBs to a user is equal to $M \times t_{s}$, where $t_{s}$ is the time needed to assign one single RB. For each user request, the scheduler needs to find and assign as many RBs as required in order to satisfy the users QoS. Since LTE-Advanced systems allow up to $500 \mathrm{RBs}$ to be assigned to a single user to exceed the $1 \mathrm{Gbps}$ requirement for IMT-A system, the time required by a scheduler to assign all the resources needed by users can become considerably high. This will result in potentially excessive delays for the scheduling tasks [49]. In order to reduce scheduling delay, a resource block organization algorithm is proposed in [48]. This strategy is based on the assignment of pre-organized RB sets depending on RB availability. The scheduler assigns sets to users as shown in Fig. 11. Based on the predefined max number of RBs and spectrum availability, RBs can be grouped into sets. Then by scheduling on a set by set basis, this scheme can help reduce the scheduling delay. However, the proposed scheme does result in the additional complexity at the scheduler due to the RB pre-organization functionality.

Since real-time traffic is more sensitive to scheduling latency than non-real time traffic, the proposed scheduling scheme of [41] prioritizes the real-time packets via two mechanisms: 1) the different dispatching frequency and 2) RB reservation.

In Fig. 12, all arrived packets are first classified into realtime (RT) and non real-time (NRT) packets by the classifier. Then RT/NRT packets are delivered into the RT/NRT queues, respectively. While RT packets in the queue can be delivered into the transmission queue for every frame, NRT packets in the NRT queue are periodically delivered every $n$ frames $(n$ : a specified integer). This scheme allows RT packets to have a greater chance to be selected by the different dispatching frequency. In addition, through the RB reservation scheme, RT packets can be transmitted over all RBs whereas NRT packets can only be transmitted over some (predefined number) RBs. However, this structure requires further investigation on how to adaptively adjust dispatching frequency and the RB reservation 
TABLE I

SUMMARY OF SCHEDULER SCHEMES

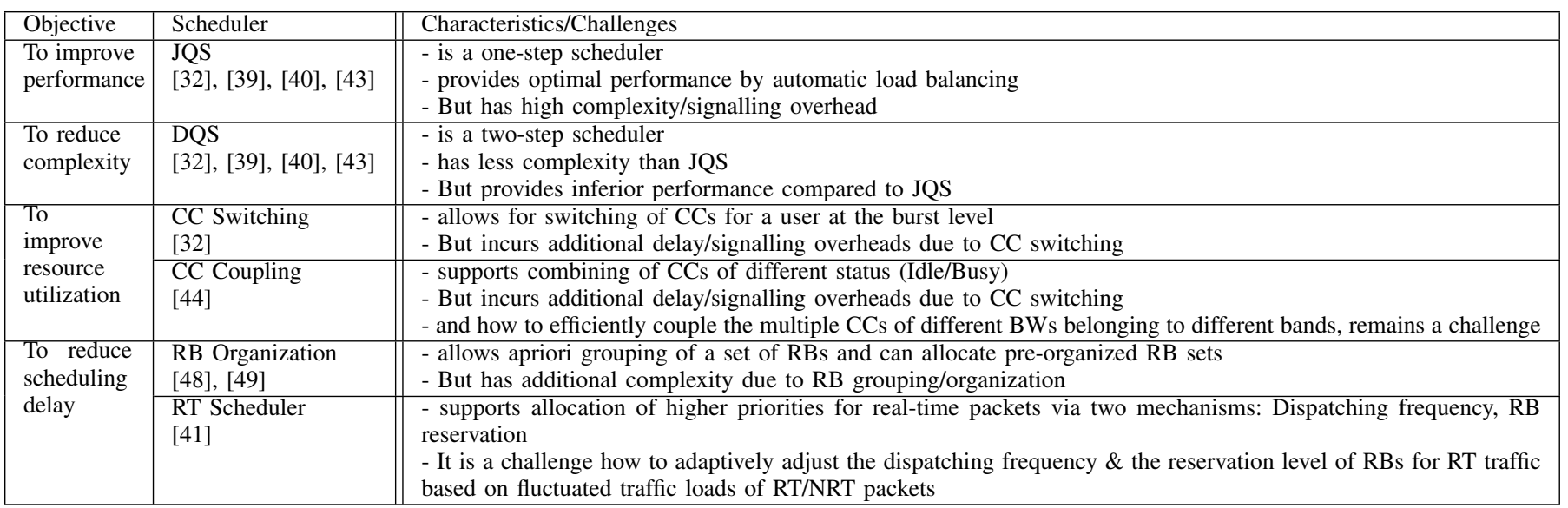

level for RT traffic during the scheduling process to achieve the best overall system performance.

TABLE I provides a summary of main characteristics of scheduling schemes considered in this section.

\section{B. CC Selection and Management}

As methods for balancing the load across CCs will affect the system performance, the following three most notable CC selection methods to address load balancing, have been the main focus of research in the literature: 1) Random Selection, 2) Circular Selection, and 3) Least Load.

In the Random Selection (RS) scheme [5], [27], [33], [36], [44], [45], CCs for each UE are chosen randomly from the available CC set by the eNB. Mobile Hashing $(\mathrm{MH})$, which relies on the output from UE's hashing algorithm, can be utilized to choose CCs randomly [27], [33], [36]. From the long term point of view, it can provide balanced load across CCs. However, at each instant, the load across CCs may not be balanced and the system may suffer from reduced spectrum utilization [27], [33].

The Circular Selection (CS) scheme used in [32], [44] selects CCs circularly for the traffic data. Compared to $R S$, it is shown that the CS scheme offers higher throughput and better coverage performance due to better balancing of traffic load over multiple CCs [44]. However, when the users traffic packet sizes are significantly different, the efficiency of this scheme can be decreased.

In the Least Load $(L L)$ rule [32], [36], [40], the scheduler allocates the users' packets to each CC according to the current traffic load of CCs. Since the packets are always allocated to the $\mathrm{CC}$ with the lowest traffic load, better load balancing across the CCs can be expected from this scheme compared with those rules (e.g. $R S$ and $C S$ ) that do not consider the system state information [44]. $L L$ rule can be expressed as follows.

$$
i^{*}=\arg \min _{i}\left(\frac{1}{B_{i}} \sum_{k=1}^{N} L\left(Q_{k, i}\right)\right)
$$

where $L\left(Q_{k, i}\right)$ is the queue length of the $k^{t h}$ user on the $i^{\text {th }} \mathrm{CC}$ and $B_{i}$ is the bandwidth of the $i^{\text {th }} \mathrm{CC}$. When the bandwidth of CCs is different, it should be considered as well as the queue length. By better balancing the load [32], [44], this scheme leads to better user fairness [36]. In [27], with the scenario of co-existing LTE-Advanced and LTE UEs, $L L$ provides a higher throughput for the LTE UEs, but lower throughput for the LTE-Advanced UEs, as compared with $R S$. This is because $L L$ distributes the LTE UEs evenly on all CCs, therefore they can get more resources than with $R S$, and hence the higher throughput. At the same time, the throughput for LTE-Advanced UEs is reduced, as fewer resources are left for them. However, in terms of average cell throughput and coverage performance, it is shown that $L L$ is always better than the $R S$ method.

Although the $L L$ scheme considers the channel characteristics based on transmission rate and the queue length of users having the data to transmit, its efficiency can be reduced depending on variations of channel quality over time. That is, the best $\mathrm{CC}$ at a given time may not be the best choice for the future traffic. In order to overcome this, in [32], the modified $L L(M-L L)$ approach using the estimated future average transmission rate is proposed and is expressed as

$$
i^{*}=\arg \min _{i}\left(\frac{1}{B_{i}} \sum_{k=1}^{N} \frac{L\left(Q_{k, i}\right)}{{R_{k}^{T}}^{T}}\right)
$$

where $\overline{R_{k}{ }^{T}}$ is the estimated average transmission rate of the $k^{t h}$ user in the next $T$ frames. While benefits highly depend on the accuracy of estimation of the average user rates, this approach could lead to higher complexity.

Although the aforementioned CC selection methods focus on load balancing to improve performance, it is worth noting that traffic load balancing alone is not enough to guarantee good system performance. For the inter-band aggregation, due to the different channel characteristics, the coverage will vary from CCs to CCs. Thus, the number of CCs over which users can be scheduled will be variable [52] i.e. users located on the cell-edge will have access to fewer CCs than those closer to cell-centre. In order to optimize the performance of inter- 
band CA, the CC selection scheme should not only take the traffic load, but also the radio channel characteristics into consideration as proposed in: inter-band carrier switch method [50], [51], RSRP based method [5], and G-factor based method [30].

In the method of inter-band carrier switch, the scenario where the operator has spectrum allocations in the $2 \mathrm{GHz}$ band for exclusive use, as well as allocations in the $5 \mathrm{GHz}$ for sharing by multiple operators is considered. Considering better quality of lower frequency carriers, the user arriving into the system is firstly allocated to the $2 \mathrm{GHz}$ band. Then, the load is checked in both bands for the load balancing; if the load in the $2 \mathrm{GHz}$ is higher than in the $5 \mathrm{GHz}$, the user with the highest CQI in the $2 \mathrm{GHz}$ will be moved to the $5 \mathrm{GHz}$ band. In situations of higher network loads, the users with the lower CQIs are allocated to the $2 \mathrm{GHz}$ band while the users with higher CQIs are allocated on the $5 \mathrm{GHz}$. The simulation results show that this scheme improves the throughput performance compared with the scheme without inter-band carrier switching. However, the proposed inter-band carrier switch scheme has the limitation that a user can be allocated CCs in the same band at the same time although it can be switched to CCs in other bands. In addition, switching the carriers could lead to the increased complexity and delay.

In [5], the RSRP based CC selection scheme is proposed addressing load balancing over CCs with the different channel characteristics. It assigns the better $\mathrm{CC}$ to the UE whose average data rate is relatively small to improve the fairness. The proposed scheme is expressed as follows.

$$
k^{*}=\arg \max _{k}\left(\frac{r s r p_{k}^{i} / \overline{R_{k}}}{\sum_{k} r s r p_{k}^{i} / \overline{R_{k}}}\right)
$$

where $r s r p_{k}^{i}$ is the channel quality on the $i^{\text {th }} \mathrm{CC}$ from the $k^{t h}$ user. $\overline{R_{k}}$ denotes the average data rate of the $k^{t h}$ user. In the simulations with real time video streaming setting, it is shown that the proposed scheme outperforms $R S$ and $L L$ schemes in terms of throughput, coverage, packet loss rate and fairness.

In [30], a geometry (G-) factor based carrier selection algorithm is proposed. Based on the threshold of G-factor, the cell-edge users are identified. For LTE users, cell-edge users are assigned to the carrier with better coverage (lowfrequency carrier) to improve the coverage performance, while other users are assigned to the carrier with the least load to balance the load on each carrier. LTE-Advanced users are assigned on all CCs. It is shown that this scheme is effective in scenarios where there are large variations in Gfactor distributions of carriers and it can significantly improve the coverage performance at the expense of marginal average user throughput loss compared with the $L L$ scheme [30].

While multiple CCs can be allocated to UEs, the use of SCCs (Secondary CCs) could be dynamically managed as mentioned in Section II. In [22], two CC management schemes for SCCs are proposed: absolute policy and relative policy. In the absolute policy, a $\mathrm{CC}$ is added or removed when signal quality of the $\mathrm{CC}$ is higher or lower than a certain threshold, respectively. In the relative policy, when signal quality of a CC is offset higher/lower than that of PCC, the CC is added/removed, respectively. It is assumed that only one CC is selected for SCC addition and all configured

TABLE II

SUMMARY OF CC SELECTION AND MANAGEMENT ALGORITHMS

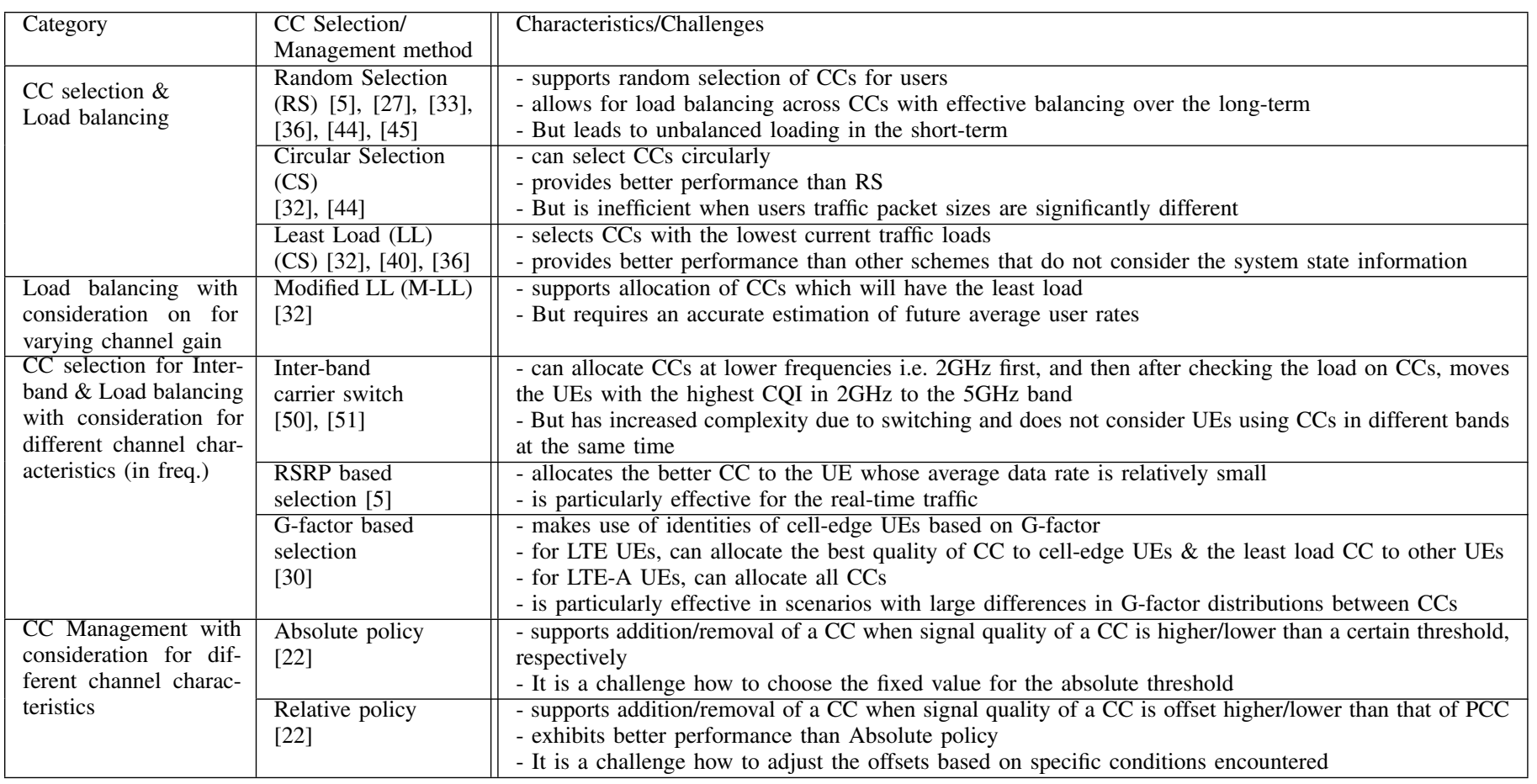


SCCs in candidate CCs of removing should be removed. Two different scenarios (scenario 1 and scenario 3) in Fig. 5 are chosen to evaluate the performance of the proposed algorithm. Simulation results show that for both schemes, the user throughput is generally higher in scenario 3 than scenario 1 , indicating effectiveness of scenario 3 type of deployment. In scenario 3 , nearly $70 \%$ of users are not really benefiting by $\mathrm{CA}$ which gives evidence to the necessity of eliminating unnecessary CCs by CC management. Furthermore, it is also shown that relative policy provides better performance than the absolute policy due to its versatility in different scenarios. Although it is pointed out that choosing a fixed threshold for absolute policy is extremely difficult, the offsets in relative policy are also required to be adjusted based on specific conditions encountered such as traffic load, carrier frequency, and bandwidth in each $\mathrm{CC}$, in real networks.

The CC selection and management schemes introduced in this section are summarized in TABLE II.

\section{RB Assignment}

Once the users are assigned onto certain $\mathrm{CC}(\mathrm{s})$, the assignment of resource blocks in each $\mathrm{CC}$ is carried out.

For RB scheduling algorithms, throughput, fairness, the trade-off between throughput and fairness and the delay performance are considered as target objectives in the literature. Especially for LTE-A system which is capable of the interband CA and allows coexistence with LTE UEs, the fairness becomes a serious issue. When inter-band CA is applied, UEs in different locations will have different number of accessible CCs. Thus, fairness between UEs of different locations should be carefully considered as well as the traditional fairness between users with different throughputs. In addition, fairness between users with different aggregation capabilities becomes a challenge. In this section, the proposed RB assignment schemes in the literature are described and later summarized in TABLE III.

As the basic scheduling scheme, Round Robin $(R R)$ is chosen as the benchmark for comparison of the performance gains of algorithms in [40], [43], [44], [45].

The Proportional Fair $(P F)$ scheme is also selected as benchmark in many works such as [4], [27], [36] due to its simplicity and good performance. Since $P F$ is aware of the channel condition for each user, it can exploit the multi-user diversity. This method allocates the $j^{\text {th }} \mathrm{RB}$ of the $i^{\text {th }} \mathrm{CC}$ to the user $k^{*}$ who maximizes its instantaneous data rate over its average data rate.

$$
k^{*}=\arg \max _{k} \frac{R_{k}(i, j, s)}{\overline{R_{k}}(i)}
$$

$R_{k}(i, j, s)$ is the instantaneous transmission rate on the $\mathrm{RB}$ $j$ of the CC $i$ for the user $k$ at the time slot $s$ and $\overline{R_{k}}(i)$ is the average delivered throughput from the $i^{\text {th }} \mathrm{CC}$ for that user in the past. In the long term, this scheduler could achieve fairness among all active users in each $\mathrm{CC}$, given that same fading statistics are assumed [53].

When CCs in different bands are given, the UEs will have different number of accessible CCs depending on their locations. In [52], In order to achieve better fairness among users, a user grouping $P F$ algorithm $(U G-P F)$ is proposed. In this scheme, with a certain threshold of path-loss, coverage of each CC is determined. Then, the users are partitioned into the specific groups, $M_{g}$, based on the number of available $\mathrm{CCs}$ that the users can be scheduled on from their location (i.e. $g$ is the number of carriers that the users in group $M_{g}$ can access). The $U G-P F$ scheduling can be described in Eq.(10).

$$
k^{*}=\arg \max _{k \in M}\left\{\frac{R_{k}(i, j, s)}{\overline{R_{k}}} \times \beta\right\}, \beta=\frac{R^{2}}{R_{g}{ }^{2}}, k \in M_{g}
$$

where $R$ is the distance from the $\mathrm{BS}$ and the user, $R_{g}$ is the coverage radius of $g^{t h} \mathrm{CC}$. Based on the weight factor $\beta$, the user group at the cell-edge has the advantage of accessing RBs in the CCs of lower frequencies because user group in the cellcenter is able to access the CCs of higher frequencies. With this scheme, the users in poor channel conditions can obtain throughput improvement and better fairness among users is achieved compared with the conventional $P F$ algorithm [35]. However, allocating a lot of RBs to users with poor channels can cause some degradation of the average cell throughput. The method to attain a good trade-off between allocation fairness and system throughput remains an open study item.

Since LTE UEs are expected to coexist with LTE-A UEs, fairness between LTE UEs and LTE-A UEs needs to be guaranteed by the LTE-A system. However, in the $P F$ scheme, LTE-Advanced user can get $L$ times the resources than a LTE user, where $L$ is the total number of aggregated $\mathrm{CCs}$ in the system. In order to improve the fairness between LTE and LTE-A users, Cross-CC PF is proposed in [27], [36].

$$
k^{*}=\arg \max _{k} \frac{R_{k}(i, j, s)}{\sum_{i=1}^{L} \overline{R_{k}}(i)}
$$

In this method, exchange of information on the past user throughput on each $\mathrm{CC}$ is required. Taking the past user throughput over all aggregated CCs makes the scheduling metric of LTE-Advanced users smaller compared with that in Eq.(9). The formulation in Eq.(11) results in better fairness to LTE users. In [27], [36], it is shown that Cross-CC PF improves the average user throughputs for LTE users thus achieving better fairness and coverage performance with no degradation in the average cell throughput. In [42], it is proved that Cross-CC $P F$ is the optimal scheduler to maximize the sum of the logarithmic user throughputs once the CC assignment is predetermined for a user.

As pointed out earlier, a challenge for $U G-P F$ scheme is how to obtain a good trade-off between throughput and fairness. In [54], two tunable parameters are introduced in PF scheme to adjust the trade-off between throughput and fairness. In [42], in order to adjust the level of fairness between UEs with different aggregation capabilities and fairness between UEs with different channel conditions, Generalized PF scheme based on Cross-CC PF is proposed as shown in Eq.(12). 


$$
k^{*}=\arg \max _{k} \frac{\alpha(k) \times R_{k}(i, j, s)}{\left(\sum_{i=1}^{L} \overline{R_{k}}(i)\right)^{\beta}}
$$

In order to adjust the fairness between different user categories, the linear weighting factor $\alpha$ in the numerator is used. $\alpha>1$ favours the LTE users as compared with multi-CC LTEA users, and vice versa. It also uses the exponential weighting factor $\beta$ in the denominator to adjust the fairness among users with different average throughput. $\beta<1$ prioritizes the users with high throughput similar to the maximum rate scheduler and $\beta>1$ favours the poor users towards an equal throughput scheduler. Depending on the settings of $\alpha$ and $\beta$, the system performance can be varied.

From the delay perspective, $P F$ scheme is not an attractive scheme to be used particularly for real-time services [41]. In [5], in order to make up for this shortcoming of $P F$, Modified Largest Weighted Delay First $(M-L W D F)$ is proposed. The terms for packet loss ratio and delay are additionally added into $P F$ and the scheme is expressed by

$$
k^{*}=\arg \max _{k}\left\{\frac{-\log \left(\delta_{k}\right) R_{k}(i, j, s) \omega_{k}(s)}{T_{k} \overline{R_{k}}}\right\}
$$

where $\delta_{k}$ and $T_{k}$ are the maximum tolerable packet loss ratio and delay of packets, respectively. $\omega_{k}(s)$ represents the actual flow delay in the buffer at the time slot $s$.

In [29], the concept of user grouping is utilized to achieve the delay fairness among users and the packet fragmentation approach is proposed. The arriving packets belonging to various groups are first buffered into their group queues, respectively. Then, the packets are partitioned with several fragments (i.e. sub-packets), each of an appropriate size, and are distributed on available $\mathrm{CCs}$ for parallel transmission simultaneously. By utilizing available $\mathrm{CC}$ effectively, the proposed scheme shows better performances in terms of the delay in each group.

\section{Joint resource allocation}

It is proven [42] that Cross-CC PF is the optimal scheduling scheme for a given $\mathrm{CC}$ selection scheme. However, the joint resource allocation in Eq.(1) shows a better performance than the two step resource allocation (i.e. CC selection and RB assignment) strategy although it has a very high computational complexity for large values of $K, N$, and $M$.

In [39], Minimizing System Utility Loss (MSUL) algorithm is proposed as a suboptimal algorithm to reduce the high computational complexity in solving the optimization problem in Eq.(1). It divides the optimization problem into a number of sub problems for each $\mathrm{CC}$, where each $\mathrm{CC}$ optimizes its $\mathrm{RB}$ allocation independently. For each $\mathrm{CC}$, the $\mathrm{RB}$ is allocated to users which achieve the maximum utility on this RB. The utility of the $k^{t h}$ user on the $j^{t h} \mathrm{RB}$ of the $i^{t h} \mathrm{CC}$ is defined by the ratio of the instantaneous throughput of the user $k$ on the $j^{\text {th }} \mathrm{RB}$ to the average aggregated throughput of the user $k$. MSUL first ignores the difference of user's CA capability and supposes that all users can access the whole CCs. Then each $\mathrm{RB}$ in every $\mathrm{CC}$ is assigned to the user that can reach the maximum utility metric. After the RB assignment, an iterative resource adjustment algorithm is performed to meet the CA capability requirement for users. In each iteration, the algorithm selects non-CA capable users allocated on multiple $\mathrm{CCs}$ and release the resource block of the minimum system utility for reassignment. Simulation results show that $M S U L$ outperforms the Cross-CC PF algorithm in terms of the sum of the logarithmic of user throughput. By jointly scheduling CCs and RBs, MSUL exploits each CC more efficiently than Cross-CC PF.

TABLE III

SUMMARY OF PROPOSED RB SCHEDULING ALGORITHMS

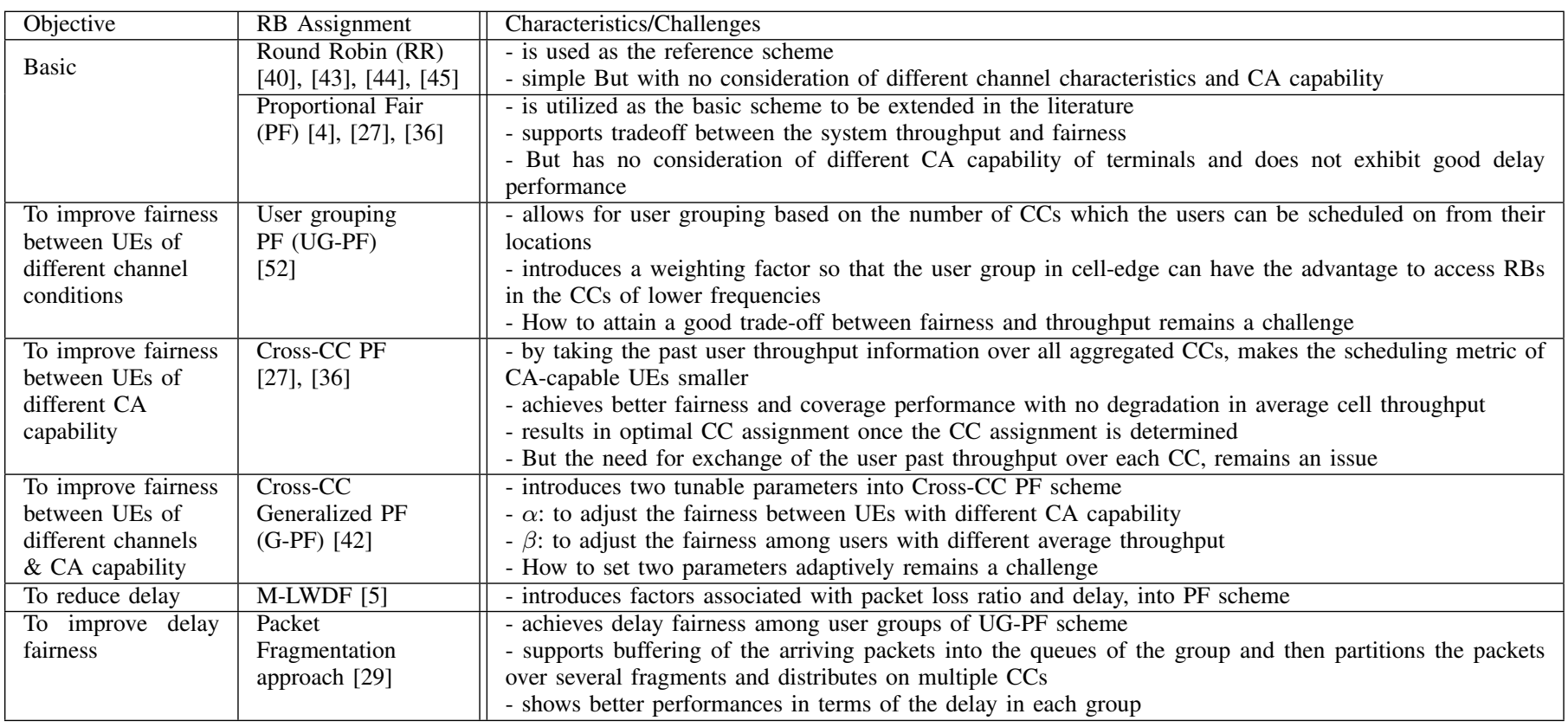




\section{E. Resource allocation for Uplink}

Although aforementioned resource allocation schemes can be applied equally on both DL and UL, allocation for the UL is required to meet the additional constraint on transmission power. In this section, the proposed resource allocation algorithms for UL considering the transmission power limit in the literature are reviewed. The summary of the algorithms described can be found in TABLE IV.

In [35], for resource allocation in the UL, users are categorized as power-limited and non-power-limited based on the path loss. In [26], a CC allocation scheme is proposed for power-limited and non-power-limited users. Assuming only two contiguous CCs available, only one $\mathrm{CC}$ is assigned to the power-limited users, while both $\mathrm{CCs}$ are assigned to non-power-limited users. System performance for different values of the path-loss threshold setting (to distinguish between power-limited and non-power-limited users) is evaluated in terms of average and cell edge user throughput. The simulation result shows that the path-loss threshold setting affects the performance significantly. If the threshold is set to be high, the average user throughput decreases, while the cell edge user throughput remains steady. This is because most LTE-A UEs, including power-limited and some non-power-limited UEs operating not close to their maximum transmission power are only assigned on one $\mathrm{CC}$ and this leads to non-power-limited LTE-A UEs not being able to benefit from the advantages of transmission bandwidth expansion afforded by CA. On the other hand, for the low setting of the threshold value, not only non-powerlimited but also some power-limited LTE-Advanced UEs are assigned on both CCs. Then, the power-limited cell edge LTE-A UEs will experience performance loss from being scheduled over multiple CCs due to further reduction of maximum UE transmission power. Therefore, both average and cell edge user throughput decrease if the threshold is set low. Since efficient methods to set path-loss threshold are required, in [26], a threshold calculation method based on the 95th-percentile user path loss in the corresponding cell, is proposed as shown in Eq.(14). The effects of increased PAPR and inter-modulation due to transmission over multiple CCs are included as the power back-off, $P_{\text {backoff } f}$.

$$
L_{\text {threshold }}=L_{95 \%}-\frac{10 \log _{10}(K)+P_{\text {backoff }}}{\alpha}
$$

where $L_{95 \%}$ is the estimated 95th-percentile user path loss in the corresponding cell (not CC-specific since only contiguous $\mathrm{CA}$ is assumed) and $K$ is the total number of allocated $\mathrm{CCs}$ for user. $\alpha$ is the CC-specific power control parameter and assumed to be the same for all CCs. Via simulations, it is shown that the proposed path-loss threshold based CC selection algorithm outperforms blind CC selection algorithm which allocates all LTE-Advanced UEs on all CCs, in terms of average and cell edge user throughput. However, the scheme is only applicable to intra-band CA scenario since it is assumed that path-loss of each CC is same.

In [35], the effect of different power back-off setting is investigated. Since the back-off power setting is known to depend on a number of factors, for simplicity, it is assumed that only the number of allocated CCs affects the back-off power setting and there is no back-off power if a UE is scheduled only one CC. Since cell edge LTE-Advanced UEs are assigned to only one $\mathrm{CC}$, the coverage performance is not influenced by the back-off power setting. However, the average throughput of LTE-Advanced UEs decreases with the increase of back-off power setting. It is because less LTE-Advanced UEs will be assigned to multiple CCs for the case of higher power back-off. Through simulation results, it is shown that the value of power back-off greatly impacts the $\mathrm{CC}$ selection of LTE-Advanced UEs.

Under the assumption of equal power allocation among subcarriers, the limitation of the maximum transmission power for uplink scheduling can decide the maximum number of supportable resource blocks for a user in order to satisfy the target SINR for the assigned RBs. A resource allocation algorithm considering the limitations on the maximum number of supportable RBs is proposed in [55]. Based on the SINR of all users on all RBs, authors formulate a priority metric matrix and allocate the RBs to users with aim of maximizing the priority sum with the constraint of maximum number of supportable RBs. The priority metric is defined as a function of SINR of the user on each RB. Simulation results indicate that the proposed algorithm improves the cell-edge user throughputs with no degradation of the average user throughput.

Since the uplink signalling for the feedback will contribute to a large amount of uplink overhead, in [31], HARQ bundling, CQI compression, and hybrid of the two methods are proposed as feedback reduction schemes. While non-CA operation generates the least overhead compared with the reduction schemes, the second highest reduction can be obtained by combining HARQ bundling with CQI compression. CQI compression on its own, performs better than HARQ bundling. Depending on the path loss value experienced by UEs, the reduction technique can be decided. However, users with reduced feedback experience lower achievable throughputs due to less accurate feedback information. Thus, combining HARQ bundling with CQI compression provides the worst performance while it has the best reduction performance. Since operating on only one $\mathrm{CC}$ generates the least overhead, it is recommended to schedule a single $\mathrm{CC}$ for the user with very poor channel quality.

When the feedback reduction techniques are used, the users will sacrifice their throughput due to the less accurate feedbacks. In fact, users with poor channel quality are already in a disadvantageous situation. It is therefore preferable not to further decrease their performance with feedback reduction techniques. In order to maintain the cell-edge user throughput, a weighted PF scheduler is proposed based on the Cross-CC $P F$ algorithm in [31] with a metric expressed in Eq.(15).

$$
k^{*}=\arg \max _{k}\left\{\frac{R_{k}(i, j, s)}{\sum_{i} \overline{R_{k}}(i)} \prod_{x=1}^{P} \beta_{x}{ }^{w_{x}}\right\}
$$

where $\beta_{x}$ is the weighting factor for $P$ kinds of overhead 
TABLE IV

SUMMARY OF RESOURCE ALGORITHMS FOR UPLINK

\begin{tabular}{|c|c|c|}
\hline Objective & Allocation algorithm & Characteristics/Challenges \\
\hline \multirow[t]{4}{*}{$\begin{array}{l}\text { To improve } \\
\text { fairness } \\
\text { between UEs } \\
\text { of different } \\
\text { channel } \\
\text { conditions }\end{array}$} & $\begin{array}{l}\text { Path-loss based } \\
\text { CC Selection [26] }\end{array}$ & $\begin{array}{l}\text { - allows separation of users into power-limited and non-power-limited } \\
\text { - can allocate one CC to the power-limited users and all CCs to non-power-limited users } \\
\text { - also proposes the method of path-loss threshold calculation based on the 95th-percentile user path loss } \\
\text { - provides better performance than allocation of all CCs to all UEs } \\
\text { - But only considers the case of intra-band CA }\end{array}$ \\
\hline & $\begin{array}{l}\text { RB scheduling with } \\
\text { max RB number } \\
\text { limit [55] }\end{array}$ & $\begin{array}{l}\text { - converts limit of the maximum transmission power into the maximum number of RBs } \\
\text { - proposes a priority metric matrix and allocates RBs to users to maximize the priority sum } \\
\text { - But only considers the case of equal power allocation among subcarriers }\end{array}$ \\
\hline & $\begin{array}{l}\text { Feedback reduction } \\
\text { scheme [31] }\end{array}$ & $\begin{array}{l}\text { - proposes HARQ bundling, CQI compression, and hybrid of the two methods } \\
\text { - proposes a weighted PF scheme for the users at the cell-edge } \\
\text { - How to set the path-loss threshold to decide a proper reduction technique and how to set the weights for } \\
\text { different feedback reduction techniques remain challenging }\end{array}$ \\
\hline & $\begin{array}{l}\text { User grouping } \\
\text { resource allocation } \\
\text { [56] }\end{array}$ & $\begin{array}{l}\text { - UEs are spatially grouping [57], [58] } \\
\text { - Only one user in each group sends feedback CQI on behalf of the entire group } \\
\text { - supports prioritization of the edge UE groups for resource allocation } \\
\text { - uses PF scheme for inter-group/intra-group scheduling in the time and frequency domains } \\
\text { - But is only suitable for the low-speed mobile UEs }\end{array}$ \\
\hline
\end{tabular}

reduction technique and $w_{x}$ equals 1 if the corresponding technique is used, otherwise it equals zero. The performance of this algorithm is evaluated in the scenario where there are $4 \mathrm{CCs}$ with $10 \mathrm{MHz}$ at $2 \mathrm{GHz}$ and $50 \%$ of the users use the feedback reduction techniques. It is observed that there is a continuous loss in average cell throughput by increasing the weighting factor. This is because the good users are getting less and less of the resources for transmission. In terms of coverage performance, increasing $\beta$ up to a certain value will provide gains, as poor users are prioritized and get more resources. However, a further increase of the value beyond this level will result in reduced coverage performance. Analysis also shows that increasing the weighting factor will cause the decrease in the frequency domain packet scheduling gain due to scheduling of users with poor channel quality. When the loss in frequency domain diversity is larger than the gain due to additional transmission resources, even the coverage performance will decrease.

In [56], a combined CC selection and RB scheduling algorithm is proposed and an investigation on the performance of cell-edge UEs is carried out. After UEs are spatiallygrouped, only one representative UE from a group is chosen to feedback CQI to the eNB on behalf of the entire group [57]. User grouping can be performed based on the normalized covariance, the distance between the UE and the standard deviation of the shadow fading [57], [58]. Then, edge UE groups having the least accessible resources are prioritized for resource allocation. Spectrum resources are scheduled to the groups based on inter-group and intra-group PF scheduling in the time and frequency domains, respectively to resolve resource contention. The proposed scheme outperforms the channel-blind Round Robin and opportunistic CA, in terms of average and cell-edge user throughputs. However, the proposed scheme is only suitable for low-speed mobile UEs. When the variation in channel conditions is drastic, the uplink CQI feedback information becomes irrelevant for scheduling even within a shorter interval. Thus, design of efficient uplink resource allocation schemes for fast moving users remains an open research topic.

\section{Conclusion and Future Research Directions}

Carrier aggregation is a powerful feature that enables flexible and efficient utilization of frequency resources ultimately resulting in significantly improved user data rates although rate of increase slows down at higher traffic loads. However, the introduction of CA is immediately followed by the decision on how many bands and which bands should be used in order to satisfy the requirements under different constraints. This imposes several challenges to the design of RRM mechanisms for CA based systems.

One significant aspect is the requirement for novel $\mathrm{CC}$ selection methods. Strategies for optimal selection of CCs for UEs, could help satisfy the QoS requirements of various traffic classes while the high system throughput and the fairness among different category of UEs (CA capability and channel conditions) can be assured. For the use of multiple CCs, since UEs need to estimate and report the channel quality information back to the eNB, the number of CCs assigned to UEs should be as less as possible considering the signal processing complexity and the power savings at the UE. In order to reduce overheads associated with feedback signalling, feedback reduction techniques have been proposed in the literature. However, the proposed methods result in reductions in the amount of feedback information at the expense of less accurate feedback information, leading to degradation in system performance. Therefore, the application of signalling overhead reduction methods should be linked to and take account of aforementioned trade-offs. In most CC selection algorithms proposed so far, inter-carrier load balancing is considered as the metric of choice due to achievable gains in spectrum utilization. The performance of the $\mathrm{CC}$ selection schemes are also subject to the deployment scenario considered.

After selecting the CCs for UEs, RB scheduling allocates available resource blocks to UEs. It is shown that good RB scheduling schemes can take advantage of the frequency diversity, which leads to increase in the spectral efficiency.

In most studies, resource allocation issue is decoupled into $\mathrm{CC}$ selection and $\mathrm{RBs}$ scheduling in order to reduce implementation complexity. In this case, however, if $\mathrm{CCs}$ 
are chosen without due consideration of the channel quality, good performance from the scheduler should not be expected. Thus, CCs selection and RB scheduling algorithms need to be considered together. Since joint CC selection and RB scheduling algorithm can have better performance compared with any decoupled $\mathrm{CC}$ selection and $\mathrm{RB}$ scheduling approaches, design of joint allocation algorithms with reasonable levels of complexity needs to be further investigated.

Depending on the deployment scenario, spectrum availability, and the device capability, the types of carrier aggregation could be determined. For the inter-band CA, different channel characteristics and transmission performance need to be considered in designing the $\mathrm{CC}$ selection schemes. In addition, the joint multiple component carrier resource allocation and adaptive adjustment of transmission parameters (e.g. transmission power, modulation, and coding schemes) for different $\mathrm{CCs}$ is still considered an open research topic. In the scenario of intra-band contiguous CA, the subcarriers in the guard bands between the CCs can be used for transmission to increase the spectral efficiency. The methods to use the guard bands could be further investigated to enhance the spectrum utilization.

The packet delay performance is considered as one of the important performance metrics to fulfil the QoS goals of high data rates, low latency of real-time applications. Typically, efficient scheduling schemes of available resources are characterized by higher delays, while simple scheduling algorithms usually waste the system resources. Although there are some existing studies aimed at improving the delay performance, more research on the delay aspect is warranted considering the application delay requirements in the next generation mobile systems.

This article has provided a literature survey on state of the art RRM schemes for CA in LTE-Advanced and has also outlined areas requiring further research and new solutions. Support of very-high-data-rates within both contiguous and non contiguous spectrum bands, via carrier aggregation is expected to continue to be one of the most important techniques in the next generation telecommunication systems.

\section{ACKNOWLEDGMENT}

This work is performed as part of OneFIT (www.ictonefit.eu) and ACROPOLIS (www.ict-acropolis.eu) projects. The projects are supported by the European Community's Seventh Framework Program (FP7).

\section{REFERENCES}

[1] ITU-R, "Background on IMT-Advanced," Tech. Rep. IMT-ADV/1-E, May 2008.

[2] ITU-R, "Requirements Related to Technical Performance for IMTAdvanced Radio Interface(s)," Tech. Rep. M.2134, Dec. 2008.

[3] ITU-R, Radio Regulations. No. V. 4, 2012.

[4] A. Osseiran, M. Xiao, and S. Ben Slimane, "Network coding in wireless communications," in Mobile and Wireless Communications for IMTAdvanced and Beyond, A John Wiley and Sons, Ltd, 2011.

[5] H. Tian, S. Gao, J. Zhu, and L. Chen, "Improved component carrier selection method for non-continuous carrier aggregation in lte-advanced systems," in Vehicular Technology Conference (VTC Fall), 2011 IEEE, pp. $1-5$, sept. 2011.

[6] 3GPP, "Dual-cell High Speed Downlink Packet Access (HSDPA) operation," TR 25.825, 3GPP, June 2008 .
[7] F. Haider, E. Hepsaydir, and N. Binucci, "Performance analysis of lteadvanced networks in different spectrum bands," in Wireless Advanced (WiAd), 2011, pp. $230-234$, jun 2011.

[8] 3GPP, "Feasibility study for Further Advancements for E-UTRA (LTEAdvanced)," TR 36.912, 3rd Generation Partnership Project (3GPP), June 2010 .

[9] N. Miki, M. , Iwamura, K. Y, and H. Ishii, "Ca for bandwidth extension in lte-advanced," Technical Journal, NTT DoCOMO, vol. 12, pp. $10-19$, september 2010.

[10] 3GPP, "Further Advancements of E-UTRA Physical Layer Aspects," TR 36.814, 3rd Generation Partnership Project (3GPP), Feb. 2009.

[11] R. Ratasuk, D. Tolli, and A. Ghosh, "Carrier aggregation in lteadvanced," in Vehicular Technology Conference (VTC 2010-Spring), 2010 IEEE 71st, pp. $1-5$, may 2010.

[12] Z. Shen, A. Papasakellariou, J. Montojo, D. Gerstenberger, and F. Xu, "Overview of 3gpp lte-advanced carrier aggregation for $4 \mathrm{~g}$ wireless communications," Communications Magazine, IEEE, vol. 50, pp. 122 -130 , february 2012.

[13] K. Pedersen, F. Frederiksen, C. Rosa, H. Nguyen, L. Garcia, and Y. Wang, "Carrier aggregation for lte-advanced: functionality and performance aspects," Communications Magazine, IEEE, vol. 49, pp. 89 -95 , june 2011.

[14] M. Iwamura, K. Etemad, M.-H. Fong, R. Nory, and R. Love, "Carrier aggregation framework in 3gpp lte-advanced [wimax/lte update]," Communications Magazine, IEEE, vol. 48, pp. 60 -67, august 2010.

[15] G. T. R. W. 61, "Ue categories for rel-10," TR R1-103348, 3rd Generation Partnership Project (3GPP), May 2010.

[16] G. Yuan, X. Zhang, W. Wang, and Y. Yang, "Carrier aggregation for lteadvanced mobile communication systems," Communications Magazine, IEEE, vol. 48 , pp. $88-93$, february 2010.

[17] 3GPP, "Carrier Aggregation-Base Station (BS) radio transmission and reception," TR 36.808, 3rd Generation Partnership Project (3GPP), 2012.

[18] 3GPP, "Evolved Universal Terrestrial Radio Access (E-UTRA) and Evolved Universal Terrestrial Radio Access Network (E-UTRAN); Overall description; Stage 2," TS 36.300, 3rd Generation Partnership Project (3GPP), Mar. 2012.

[19] M. Al-Shibly, M. Habaebi, and J. Chebil, "Carrier aggregation in long term evolution-advanced," in Control and System Graduate Research Colloquium (ICSGRC), 2012 IEEE, pp. 154-159, 2012.

[20] S. Parkvall, A. Furuskar, and E. Dahlman, "Evolution of lte toward imtadvanced," Communications Magazine, IEEE, vol. 49, no. 2, pp. 84-91, 2011.

[21] I. F. Akyildiz, D. M. Gutierrez-Estevez, and E. C. Reyes, "The evolution to 4G cellular systems: LTE-Advanced," Physical Communication, Aug. 2010.

[22] L. Liu, M. Li, J. Zhou, X. She, L. Chen, Y. Sagae, and M. Iwamura, "Component carrier management for carrier aggregation in lte-advanced system," in Vehicular Technology Conference (VTC Spring), 2011 IEEE $73 \mathrm{rd}$, pp. $1-6$, may 2011.

[23] T.-T. Tran, Y. Shin, and O.-S. Shin, "Overview of enabling technologies for 3gpp lte-advanced," EURASIP Journal on Wireless Communications and Networking, vol. 2012, no. 1, p. 54, 2012.

[24] L. Lindbom, R. Love, S. Krishnamurthy, C. Yao, N. Miki, and V. Chandrasekhar, "Enhanced Inter-cell Interference Coordination for Heterogeneous Networks in LTE-Advanced: A Survey," tr, , dec. 2011.

[25] M. Kottkamp, A. Roessler, and J. Schilenz, "LTE-Advanced Technology Introduction: White Paper," tr, Rohde and Schwarz, 2010.

[26] H. Wang, C. Rosa, and K. Pedersen, "Uplink component carrier selection for lte-advanced systems with carrier aggregation," in Communications (ICC), 2011 IEEE International Conference on, pp. 1-5, 2011.

[27] Y. Wang, K. Pedersen, P. Mogensen, and T. Srensen, "Resource allocation considerations for multi-carrier lte-advanced systems operating in backward compatible mode," in Personal, Indoor and Mobile Radio Communications, 2009 IEEE 20th International Symposium on, pp. 370 -374 , sept. 2009.

[28] S. Parkvall and D. Astely, "The evolution of lte towards imt-advanced," JCM, vol. 4, no. 3, pp. 146-154, 2009.

[29] Y.-L. Chung and Z. Tsai, "A quantized water-filling packet scheduling scheme for downlink transmissions in lte-advanced systems with carrier aggregation," in Software, Telecommunications and Computer Networks (SoftCOM), 2010 International Conference on, pp. 275 -279, sept. 2010.

[30] H. Wang, C. Rosa, and K. I. Pedersen, "Performance analysis of downlink inter-band carrier aggregation in lte-advanced.," in VTC Fall, pp. 1-5, IEEE, 2011

[31] Y. Wang, K. Pedersen, M. Navarro, P. Mogensen, and T. Srensen, "Uplink overhead analysis and outage protection for multi-carrier lteadvanced systems," in Personal, Indoor and Mobile Radio Communica- 
tions, 2009 IEEE 20th International Symposium on, pp. 17 -21, sept. 2009.

[32] L. Zhang, K. Zheng, W. Wang, and L. Huang, "Performance analysis on carrier scheduling schemes in the long-term evolution-advanced system with carrier aggregation," Communications, IET, vol. 5, pp. $612-619$, 252011.

[33] Y. Wang, K. Pedersen, P. Mogensen, and T. Sorensen, "Carrier load balancing methods with bursty traffic for lte-advanced systems," in Personal, Indoor and Mobile Radio Communications, 2009 IEEE 20th International Symposium on, pp. 22 -26, sept. 2009.

[34] Q. Europe, "LTE-A MC RF requirements for contiguous carriers," TR R4-091910, 3GPP TSG-RAN WG4 51, Apr. 2009.

[35] H. Wang, C. Rosa, and K. Pedersen, "Performance of uplink carrier aggregation in lte-advanced systems," in Vehicular Technology Conference Fall (VTC 2010-Fall), 2010 IEEE 72nd, pp. 1 -5, sept. 2010.

[36] Y. Wang, K. Pedersen, T. S andrensen, and P. Mogensen, "Carrier load balancing and packet scheduling for multi-carrier systems," Wireless Communications, IEEE Transactions on, vol. 9, pp. 1780 -1789, may 2010.

[37] 3GPP, "Medium Access Control (MAC) protocol specification (Release 11)," TR 36.321, 3rd Generation Partnership Project (3GPP), 2013.

[38] K. Yagyu, T. Nakamori, H. Ishii, M. Iwamura, N. Miki, T. Asai, and J. Hagiwara, "Investigation on mobility management for carrier aggregation in lte-advanced," in Vehicular Technology Conference (VTC Fall), 2011 IEEE, pp. 1 -5, sept. 2011.

[39] F. Wu, Y. Mao, S. Leng, and X. Huang, "A carrier aggregation based resource allocation scheme for pervasive wireless networks," in $D e$ pendable, Autonomic and Secure Computing (DASC), 2011 IEEE Ninth International Conference on, pp. 196 -201, dec. 2011.

[40] L. Chen, W. Chen, X. Zhang, and D. Yang, "Analysis and simulation for spectrum aggregation in lte-advanced system," in Vehicular Technology Conference Fall (VTC 2009-Fall), 2009 IEEE 70th, pp. 1 -6, sept. 2009.

[41] Y.-L. Chung, L.-J. Jang, and Z. Tsai, "An efficient downlink packet scheduling algorithm in lte-advanced systems with carrier aggregation," in Consumer Communications and Networking Conference (CCNC), 2011 IEEE, pp. $632-636$, jan. 2011.

[42] Y. Wang, K. Pedersen, T. Sorensen, and P. Mogensen, "Utility maximization in lte-advanced systems with carrier aggregation," in Vehicular Technology Conference (VTC Spring), 2011 IEEE 73rd, pp. 1 -5, may 2011.

[43] L. Zhang, Y. Y. Wang, L. Huang, H. L. Wang, and W. B. Wang, "Qos performance analysis on carrier aggregation based lte-a systems," in Wireless Mobile and Computing (CCWMC 2009), IET International Communication Conference on, pp. 253 -256, dec. 2009.

[44] L. Zhang, F. Liu, L. Huang, and W. Wang, "Traffic load balance methods in the lte-advanced system with carrier aggregation," in Communications, Circuits and Systems (ICCCAS), 2010 International Conference on, pp. $63-67$, july 2010.

[45] L. Lei and K. Zheng, "Performance evaluation of carrier aggregation for elastic traffic in lte-advanced systems," IEICE Transactions, pp. 35163519, 2009.

[46] Ofcom, "4G Capacity Gains: Report for Ofcom," tr, Real Wireless Ltd., jan. 2011.

[47] H. Holma and A. Toskala, LTE for UMTS - OFDMA and SC-FDMA Based Radio Access. Wiley Publishing, 2009.

[48] G. Galaviz, D. Covarrubias, and A. Andrade, "On a spectrum resource organization strategy for scheduling time reduction in carrier aggregated systems," Communications Letters, IEEE, vol. 15, pp. $1202-1204$, november 2011.

[49] G. Galaviz, D. Covarrubias, n. G. Andrade, and S. Villarreal, "A resource block organization strategy for scheduling in carrier aggregated systems.," EURASIP J. Wireless Comm. and Networking, vol. 2012, p. $107,2012$.

[50] F. Meucci, O. Cabral, F. Velez, A. Mihovska, and N. Prasad, "Spectrum aggregation with multi-band user allocation over two frequency bands," in Mobile WiMAX Symposium, 2009. MWS '09. IEEE, pp. $81-86$, july 2009.

[51] A. Mihovska, "Spectrum aggregation with optimal multi-band scheduling," Wireless Personal Multimedia Communications Symposia. Proceedings, vol. 2010, pp. S11-4, 2010.

[52] S. Songsong, F. Chunyan, and G. Caili, "A resource scheduling algorithm based on user grouping for lte-advanced system with carrier aggregation," in Computer Network and Multimedia Technology, 2009. CNMT 2009. International Symposium on, pp. 1 -4, jan. 2009.

[53] J. Holtzman, "Asymptotic analysis of proportional fair algorithm," in Personal, Indoor and Mobile Radio Communications, 2001 12th IEEE International Symposium on, vol. 2, pp. F-33 -F-37 vol.2, sep/oct 2001.
[54] C. Wengerter, J. Ohlhorst, and A. von Elbwart, "Fairness and throughput analysis for generalized proportional fair frequency scheduling in ofdma," in Vehicular Technology Conference, 2005. VTC 2005-Spring. 2005 IEEE 61st, vol. 3, pp. 1903 - 1907 Vol. 3, may-1 june 2005.

[55] F. Liu and Y. Liu, "Uplink scheduling for lte-advanced system," in Communication Systems (ICCS), 2010 IEEE International Conference on, pp. $316-320$, nov. 2010.

[56] R. Sivaraj, A. Pande, K. Zeng, K. Govindan, and P. Mohapatra, "Edgeprioritized channel- and traffic-aware uplink carrier aggregation in lte-advanced systems," in World of Wireless, Mobile and Multimedia Networks (WoWMoM), 2012 IEEE International Symposium on a, pp. 1 -9 , june 2012.

[57] W. Lee and D.-H. Cho, "Cqi feedback reduction based on spatial correlation in ofdma system," in Vehicular Technology Conference, 2008. VTC 2008-Fall. IEEE 68th, pp. 1 -5, sept. 2008.

[58] M. Gudmundson, "Correlation model for shadow fading in mobile radio systems," Electronics Letters, vol. 27, pp. 2145 -2146, nov. 1991. 